15 | 2017

Le corps humain saisi par le droit : entre liberté et propriété

\title{
Les utilités du corps d'autrui : le contrôle des autorités parentales et tutélaires
}

The Usages of the Body of Another: The Control Exercised by Parents and Guardians

\section{Gilles Raoul-Cormeil}

\section{OpenEdition} Journals

Édition électronique

URL : https://journals.openedition.org/crdf/555

DOI : $10.4000 /$ crdf.555

ISSN : 2264-1246

\section{Éditeur}

Presses universitaires de Caen

\section{Édition imprimée}

Date de publication : 1 novembre 2017

Pagination : 87-96

ISBN : 978-2-84133-858-0

ISSN : $1634-8842$

\section{Référence électronique}

Gilles Raoul-Cormeil, «Les utilités du corps d'autrui : le contrôle des autorités parentales et tutélaires », Cahiers de la recherche sur les droits fondamentaux [En ligne], 15 | 2017, mis en ligne le 01 octobre 2019, consulté le 15 novembre 2022. URL : http://journals.openedition.org/crdf/555; DOI : https://doi.org/10.4000/crdf.555 


\title{
Les utilités du corps d'autrui: le contrôle des autorités parentales et tutélaires
}

\author{
Gilles RAOUL-CORMEIL \\ Maître de conférences (HDR) en droit privé à l'université de Caen Normandie \\ Institut Demolombe (EA 967)
}

I. La légitimation du contrôle parental ou tutélaire dans l'ordre du Code civil

A. Fondement et technique de la protection des mineurs

B. Fondement et technique de la protection des majeurs vulnérables

II. La dégradation du contrôle parental ou tutélaire dans l'ordre du Code de la santé publique

A. L'élargissement du cercle des personnes ayant autorité

B. Le relâchement de la valeur de leur consentement

Au pays de Descartes, il n'est pas encore devenu aisé de penser la relation entre la personne et son corps.

La nature m'enseigne [...] que je ne suis pas seulement logé dans mon corps, ainsi qu'un pilote en son navire, mais, outre cela, que je lui suis conjoint très étroitement et tellement confondu et mêlé, que je compose comme un seul tout avec lui ${ }^{1}$.

Descartes admettait la dualité du corps et de l'esprit dans la mesure où la matérialité du corps peut être l'objet d'une pensée réfléchie ${ }^{2}$. Mais le philosophe concédait l'interdépendance du corps et de l'esprit dès lors que les souffrances du corps rejaillissent sur la liberté de l'esprit. Les philosophes ${ }^{3}$ ne raisonnent plus, comme Descartes, sur l'union de l'âme et du corps mais sont interrogés par les progrès de la science biomédicale et sur le bien-fondé des limites que la législation est contrainte de porter à la liberté individuelle ou au pouvoir médical ${ }^{4}$. L'introduction des articles 16 à 16-9 du Code civil régissant le corps humain a conduit les juristes à développer une théorie générale du corps humain et à prendre parti sur sa nature juridique. Rares sont les auteurs qui, dans le prolongement de la philosophie dualiste, néoplatonicienne ou cartésienne, conçoivent que le corps humain soit «une chose $»^{5}$ et

1. R. Descartes, Méditation sixième, in Méditations métaphysiques [1641], C. Adam, P. T. Tannery (éd.), Paris, J. Vrin, 1983, p. 64.

2. Dans le Cogito, ergo sum, ce moi, «l'âme par laquelle je suis ce que je suis» est «entièrement distinct du corps» (R. Descartes, Discours de la méthode, Paris, Éditions du monde moderne, 1637, quatrième partie, p. 44).

3. Voir spécialement T. Machefert, «Peut-on fonder une éthique sur la liberté? Les apories de l'individualisme dans la philosophie morale contemporaine", in La libre disposition de son corps, J.-M. Larralde (dir.), Bruxelles, Nemesis - Bruylant (Droit et justice; 88), 2009, p. 19-43.

4. Sur lequel, voir A. Catherine, Pouvoir du médecin et droits du patient. L'évolution de la relation médicale, thèse de doctorat en droit public, université de Caen Normandie, 2011.

5. Sur la nature juridique objective du corps, voir F. Zenati-Castaing, T. Revet, Manuel de droit des personnes, Paris, PUF (Droit fondamental. Manuels), 2006, leçon 8, n 275, p. 237: «La loi affirme cette nature en organisant la protection du corps par le moyen d'un droit subjectif: la proclamation, par l'article 16-1, alinéa 1, du Code civil, du droit de chacun au respect de son corps consacre un pouvoir du sujet sur son corps, ce qui requiert sa qualité de chose». Voir aussi J.-C. Galloux, «De corpore jus, premières analyses sur le statut juridique du corps humain, ses éléments et ses produits selon les lois n $94-653$ et 94-654 du 29 juillet 1994", Petites affiches, $n^{\circ} 149,14$ décembre 1994, p. 18-24, spéc. nº 29, p. 22. 
même « un bien $»^{6}$ : un objet appropriable, bien que non saisissable ${ }^{7}$. Toute personne a la libre disposition de son corps et peut accéder exclusivement à toutes ses utilités. L'analyse en termes de propriété individuelle concède volontiers l'existence de limites légales à la liberté de la personne de disposer de son corps ${ }^{8}$. Mais de nombreux juristes répugnent à placer le corps humain dans le monde des choses, l'analysant comme une " composante de la personne ${ }^{9}$. La philosophie moniste qui refuse de distinguer la personne et son corps comprend encore de nombreux partisans formés par les auteurs classiques ${ }^{10}$. D'emblée, admettons qu'il soit assez difficile de concevoir que le corps humain soit saisi comme un objet de droit distinct du sujet qui pense et qui entend exercer sur celui-ci des actes juridiques qui l'obligent à l'égard d'autrui. Obliger son corps, n'est-ce pas déjà, et surtout, s'obliger soi-même, par sa volonté? La complexité de la question doit être admise tant elle mobilise la conscience individuelle et des savoirs pluridisciplinaires pour entreprendre et mener une réflexion éclairée. La dimension philosophique, morale ou religieuse du débat est fondamentale mais elle doit être tenue pour ce qu'elle est et ne pas troubler la distinction de la personne et des choses ${ }^{11}$. Le Code civil de 1804 a consacré le concept juridique et politique de sujet de droit: la personnalité juridique est un masque porté par l'être humain en vie, de sa naissance à sa mort. La qualité de personne protège d'abord le corps humain; elle donne ensuite du sens à la libre disposition de celui-ci; elle permet enfin de limiter la volonté individuelle du sujet de droit en l'obligeant à respecter des règles d'ordre public.

La relation de la personne et de son corps devient plus aisée à concevoir lorsqu'il s'agit d'éprouver la légitimité de la décision prise par une personne sur le corps d'une autre sur laquelle elle a autorité. La distinction du corps et de la personne perd toute sa pertinence; elle n'est plus une prémisse nécessaire pour conduire la réflexion. La question se déplace et se recentre sur la légitimité du pouvoir d'exercer une autorité - parentale ou tutélaire. Cette légitimité est alors éprouvée et analysée à l'aune du bienfait ou du mal, du risque ou de l'avantage que peut en retirer la personne vulnérable, sans qu'il soit nécessaire de distinguer son corps et son esprit, son présent et son avenir, alors confondus dans la détermination de son intérêt individuel. Une personne vulnérable peut-elle seule engager son corps? À quel niveau d'altération des facultés mentales ou d'immaturité du discernement faut-il supposer que la personne ne peut plus, ou pas encore, prendre seule de décision qui l'engage en son corps. On est là dans des cas où l'autonomie de la personne à disposer d'elle-même perd une partie de son sens, où la protection juridique se manifeste par des institutions séculaires: l'autorité tutélaire d'un père, d'une mère ou d'un tiers ayant reçu du juge le titre de curateur ou de tuteur. Le concept d'autorité est décisif; c'est un élément tiers dans le débat ayant convoqué la liberté et la propriété pour expliquer la relation de la personne juridique au corps humain.

Le droit des incapacités et de l'autorité parentale ont été réécrits par les lois Carbonnier ${ }^{12}$ à une époque où le législateur n'avait pas jugé nécessaire de descendre dans le détail de la protection de la personne. Les lois récentes ${ }^{13}$ ont affronté cette difficulté sans qu'il ne résulte cependant d'harmonisation entre le droit de l'autorité parentale ou le droit tutélaire, d'une part, et le droit de la

6. F. Zenati-Castaing, T. Revet, Manuel de droit des personnes, $\mathrm{n}^{\circ} 277, \mathrm{p} .238$.

7. Voir P. Berlioz, La notion de bien, Paris, LGDJ (Bibliothèque de droit privé; 489), 2007. Voir aussi P. Berlioz, Droit des biens, Paris, Ellipses, 2014.

8. G. Cornu, Droit civil. Les personnes, $13^{\mathrm{e}}$ éd., Paris, Montchrestien (Domat droit privé), 2007, $\mathrm{n}^{\circ}$ 20, p. 39: «Le principe d'autodisposition existe positivement mais il est soumis à diverses exigences et se heurte à d'irréductibles limites».

9. F. Terré, D. Laszlo-Fenouillet, Droit civil. Les personnes, la famille, les incapacités, $6^{\circ}$ éd., Paris, Dalloz (Précis Dalloz), 1996, n 14, p. 14 (« Division»). Voir aussi F. Terré, D. Laszlo-Fenouillet, Droit civil. Les personnes: personnalité, incapacité, protection, $8^{\mathrm{e}}$ éd., Paris, Dalloz (Précis Dalloz), 2012, $\mathrm{n}^{\mathrm{o}} 14, \mathrm{p} .15$, où les auteurs expliquent que les progrès de la science, et avec eux les lois dites de bioéthique, ont rendu nécessaires des analyses nouvelles sur l'existence de la personnalité juridique, spécialement la relation de la personne et de son corps ( $\left.\mathrm{n}^{\circ} 17\right)$. À comparer avec $\mathrm{P}$. Malaurie, Droit des personnes: la protection des mineurs et des majeurs, $8^{e}$ éd., Issy-les-Moulineaux, LGDJ, 2016, $\mathrm{n}^{\circ}$ 3, p. 5-6 ("Âme et corps"), où l'auteur explique les difficultés contemporaines de la bioéthique qui, selon les circonstances, hésite entre la réification et la personnification du corps humain.

10. J. Carbonnier, Droit civil, vol. I, Les personnes, la famille, l'enfant, le couple, Paris, PUF (Quadrige. Manuels), 2004, n 198, p. 384: «Le corps humain est hors du commerce au sens de l'art. 1128 C.civ. Il a beau, à de certains égards, être senti par la personne comme quelque chose qui lui est extérieur, il est, au fond, la personne elle-même». À ces développements en gros caractères, l'auteur ajoute en petits caractères: «On peut reprocher à une telle analyse (qui défend que la personne est propriétaire de son corps) de considérer le corps humain comme une chose, alors qu'il est la personne même, et de confondre ainsi sujet et objet. Aussi l'opinion la plus répandue aujourd'hui verrait-elle plutôt dans le droit sur son propre corps un droit de la personnalité, un des droits primordiaux de l'individu». Voir aussi D. Thouvenin, «La personne et son corps: un sujet humain pas un individu biologique», Petites affiches, ${ }^{\circ}{ }^{149}, 14$ décembre 1994, p. 25-28, spéc. p. 26 : «Si le corps humain fait son entrée dans le Code civil, c'est bien comme lié à la personne [...]. Si les énoncés des nouveaux articles hésitent entre la personne et le corps, c'est bien parce que toucher au corps, c'est toucher à la personne même, ce qui se traduit par l'affirmation de l'inviolabilité du corps humain ".

11. F. Zenati-Castaing, T. Revet, Manuel de droit des personnes, $\mathrm{n}^{\circ} 276$ in fine, p. 239: "La construction du corps par la modernité n'affecte pas sa conception comme chose car le sujet n'est pas corps mais concept. La personne juridique étant purement formelle, le corps lui est extérieur. Le Code civil de 1804 ne traite pas de l'homme, mais du sujet. La reprise de la distinction romaine des personnes et des choses oppose les sujets aux objets. Le corps n'étant pas sujet, il deviendra naturellement objet lorsque le droit civil s'y intéressera. La summa divisio des personnes et des choses l'interdit d'autant moins qu'elle demeure un procédé de classement au service d'une systématisation: elle ne comporte pas la dimension philosophique, religieuse et morale qu'on lui prête aujourd'hui». Voir T. Revet, «Le corps humain est-il une chose appropriée?», Revue trimestrielle de droit civil, 2017, p. 587 sq.

12. Loi no 64-1164 du 14 décembre 1964 sur la tutelle des mineurs et l'émancipation; loi no 68-5 du 3 janvier 1968 sur le droit des incapables majeurs; loi nº 70-459 du 4 juin 1970 relative à l'autorité parentale. Sur ces trois réformes replacées dans la marche des neuf sœurs, voir J. Carbonnier,

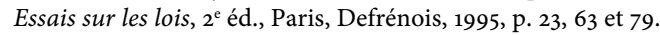

13. Loi no $2002-305$ du 4 mars 2002 relative à l'autorité parentale; loi nº $2007-308$ du 5 mars 2007 sur la réforme de la protection juridique des majeurs. 
santé publique, d'autre part. Les incohérences du régime juridique du corps humain sont imputables à la technique formelle de la codification ${ }^{14}$ à l'épreuve de laquelle se pose le problème substantiel du choix et du sens des concepts juridiques. Ainsi, la notion de «consentement» et son contraire, la situation d'être «hors d'état de manifester sa volonté», ont des critères et des portées différentes selon qu'il s'agit de fonder la validité d'un contrat au sens de l'article 1128 du Code civil ${ }^{15}$ ou de justifier le respect de la dignité humaine en recherchant l'adhésion du patient à un acte médical ${ }^{16}$.

L'analyse du régime juridique des actes médicaux $\mathrm{du}$ mineur et du majeur protégé révèle d'autres contrastes. Par souci de clarté pédagogique et de concision de la démonstration, nous avons choisi d'exclure de cette analyse le corps humain sans vie ${ }^{17}$ ou le corps humain en gestation ${ }^{18}$. Non seulement ces corps d'autrui dépendent ici et là de cadres juridiques singuliers mais la volonté des sujets vivants et présents l'emporte généralement sur celle des personnes à venir ou décédées ab intestat. Dans tous ces cas, l'autonomie du sujet n'a pas de sens. À s'en tenir alors aux actes de soins des personnes vulnérables vivantes, mineures ou majeures protégées, la question est de savoir ce que révèle l'étude de l'intervention d'un parent ou d'un tuteur dans la décision médicale portant sur le corps de la personne vulnérable. Les règles sont obscures; elles ne permettent pas, malgré leur nombre et leur réécriture récurrente, de dégager des principes clairs et féconds; elles s'articulent mal entre elles et révèlent un paradoxe. Dans le Code civil, siège de la philosophie de l'autonomie de la volonté, la loi fonde encore la légitimité du contrôle parental ou tutélaire sur le consentement de la personne vulnérable (I). En revanche, le Code de la santé publique neutralise en grande partie cette philosophie volontariste; il accorde toute légitimité à l'autorité des médecins dont la science et la profession fondent le pouvoir et la responsabilité de la décision médicale. Dans ce contexte très réglementé, l'intervention d'une tierce personne dans le colloque singulier du médecin et de son patient est suspecte et mise à distance (II).

\section{La légitimation du contrôle parental ou tutélaire dans l'ordre du Code civil}

Dans l'ordre du Code civil, la protection des mineurs et celle des majeurs protégés obéissent à des règles distinctes pour éviter l'infantilisation de ces derniers. L'intervention d'un parent ou d'un tiers repose sur des philosophies différentes. Au commencement de sa vie, l'enfant mineur a besoin d'être protégé par ses parents et qui mieux que ceux qui ont provoqué son existence peuvent le prendre en charge. La loi fait confiance aux parents: elle leur accorde des devoirs et des prérogatives. En revanche, il n'est ni naturel ni philosophiquement souhaitable qu'un adulte soit assujetti à autrui, ou, au contraire, ait tout pouvoir sur lui, dans le gouvernement de sa vie personnelle. Depuis les Lumières, la loi présume le libre arbitre. Certes, par exception, la loi répond au besoin d'aide aux personnes vulnérables en soumettant les personnes en charge de la protection juridique à diverses obligations : respecter l'autonomie du sujet protégé et solliciter l'autorisation du juge dans les cas les plus graves. L'intervention d'un parent ou d'un tiers est ainsi soumise à une pluralité de techniques juridiques dont l'agencement est propre à la protection des mineurs $(\mathrm{A})$ et à celle des majeurs vulnérables placés sous mesure de protection juridique (B).

\section{A. Fondement et technique de la protection des mineurs}

Pour les mineurs, la loi définit l'autorité parentale comme un ensemble de devoirs et de prérogatives ayant pour finalité l'intérêt de l'enfant ${ }^{19}$. L'article $371-1$ du Code civil oblige les parents qui exercent l'autorité parentale à associer l'enfant aux décisions qui le concernent, selon son âge et son degré de maturité. Toute décision concernant le corps du mineur est prise par le(s) parent(s) mais préparée avec l'enfant mineur, car il importe que la décision repose sur la responsabilité des parents. Si le mineur manque de maturité ou de discernement, la décision est consentie par ses parents, les deux ou l'un des deux seulement.

14. Voir déjà G. Raoul-Cormeil, «La santé dans le Code civil», Revue générale de droit médical, numéro spécial, $1^{\mathrm{er}}$ décembre 2010 , Santé et droit (Actes du colloque tenu à Caen le 15 octobre 2009), M. Couturier, A. Catherine (dir.), p. 29-51.

15. Art. 414-1 ou 1129 du Code civil, siège de la règle selon laquelle il faut être sain d'esprit pour conclure un acte juridique. Sur le défaut de consentement depuis l'ordonnance $\mathrm{n}^{\circ}$ 2016-131 du 10 février 2016 entrée en vigueur le $1^{\text {er }}$ octobre 2016, voir La réforme du droit des contrats. Commentaire article par article, T. Douville (dir.), Issy-les-Moulineaux, Gualino, 2016, spéc. p. 77 sq. Voir aussi O. Simon, «La nullité des actes juridiques pour trouble mental», Revue trimestrielle de droit civil, 1974, p. 707-738; G. Raoul-Cormeil, F. Rogue, "L'insécurité juridique tenant à l'insanité ou à l'incapacité d'une partie à l'acte notarié », Petites affiches, n 86,30 avril 2015 (111 congrès des notaires de France, «La sécurité juridique», Strasbourg, 10-13 mai 2015), p. 27-46.

16. Art. 16-3 du Code civil; art. L. 1111-4 du Code de la santé publique. Sur ces textes, voir G. Mémeteau, "À l'abordage (de l'article 16-3 du Code civil) », Petites affiches, $\mathrm{n}^{\circ} 149,14$ décembre 1994, p. 41-48; D. Thouvenin, «Les avatars de l'article 16-3, alinéa 1 du code civil (après la loi nº $99-941$ du 27 juillet 1999 portant création de la couverture maladie universelle)», Recueil Dalloz, 200o, chron., p. 485-490; A. Batteur, «Le consentement sur le corps humain en matière médicale», in La libre disposition de son corps, p. 45-69.

17. Sur le cadavre et les reliques, voir X. Labbée, "Le chef de saint Yves», Recueil Dalloz, 2006, p. 1833. Sur la réification inévitable du corps humain et sur le statut du cadavre en droit français saisi comme une chose publique humaine, voir F. Bellivier, Droit des personnes, Issy-les-Moulineaux, LGDJ - Lextenso éditions (Domat droit privé), 2015, nº 207 sq., p. 191-202.

18. L'embryon in vitro ou in vivo. Voir dans ce volume C. Chaput-Le Bars, T. Chartrin, G. Raoul-Cormeil, «Naissances blanches - le deuil périnatal entre propriété du corps de l'enfant et liberté du sujet».

19. Voir P. Bonfils, A. Gouttenoire, Droit des mineurs, $2^{\mathrm{e}}$ éd., Paris, Dalloz (Précis. Droit privé), 2014, $\mathrm{n}^{\circ}$ 1244 sq., p. 749-754; J. Poirret, La représentation légale du mineur sous autorité parentale, thèse de doctorat, université Paris 12 - Paris-Est-Créteil-Val-de-Marne, 2011. 
La représentation fait place à l'assistance lorsque le mineur fait preuve d'une maturité suffisante ${ }^{20}$.

Le Code civil prend en considération la gravité de la décision qui concerne le corps ou la vie personnelle de l'enfant mineur. À cet effet, l'article 372-2 du Code civil distingue les actes usuels et les autres. Les premiers peuvent être pris par un parent seul, car il est présumé avoir reçu l'accord de l'autre. La règle protège les tiers qui peuvent se retrancher derrière cette présomption légale d'accord des père et mère. En revanche, pour les décisions qui ne sont pas usuelles, la décision doit être prise par les deux parents. La qualification de décision usuelle n'est pas toujours aisée à déterminer: l'acte usuel repose sur l'habitude ou le caractère ordinaire de la décision. Le médecin qui ne recherche pas le consentement de l'autre parent parce qu'il s'est trompé sur la qualification d'acte usuel s'expose à des sanctions disciplinaires ${ }^{21}$.

Les textes du Code civil nous portent à tirer trois leçons.

Leçon $\mathrm{n}^{\circ}$ 1. Le législateur fait confiance aux parents; il ne les oblige pas à requérir une autorisation du juge aux affaires familiales. La règle ne reçoit exception qu'en cas de désaccord.

Leçon $n^{\circ} 2$. Le législateur organise surtout les rapports entre les père et mère ou les parents de même sexe. En cas d'exercice conjoint de l'autorité parentale, la loi distingue la gestion concurrente (actes usuels) et la gestion conjointe (actes non usuels). En cas d'exercice unilatéral de l'autorité parentale, le parent qui ne bénéficie que d'un droit de visite et d'hébergement peut s'opposer à la décision du parent (qui exerce seul l'autorité parentale) en sollicitant le contrôle du juge aux affaires familiales.
Leçon $n^{\circ} 3$. Rien ou presque n'est dit sur l'autonomie du mineur dans le Code civil, à l'exception du droit de la biomédecine. L'argument tiré de l'autonomie du mineur est fondé sur l'article 16-3 du Code civil, introduit par la loi no $94-653$ du 29 juillet 1994 sur le corps humain. Il faut donc raisonner en termes d'incapacité d'exercice, faisant que la personne mineure est, selon son degré de maturité et de discernement, assistée ou représentée par ses père et mère. Le contrôle parental est donc ici légitime.

Toutefois, la légitimité du contrôle parental connaît deux séries d'exceptions élevées par le Code de la santé publique qu'il convient ici de relever car elles n'ignorent pas le Code civil.

D'une part, le Code de la santé publique établit des incapacités spéciales de jouissance, c'est-à-dire que la loi interdit exceptionnellement certains actes médicaux sur des mineurs dans le but de les protéger de manière absolue. Tel est le cas de la stérilisation définitive à visée thérapeutique $\mathrm{e}^{22}$ ou du don de gamètes par un enfant mineur qui n'a pas déjà engendré ${ }^{23}$. Il faudrait aussi citer l'interdiction de rechercher le consentement du mineur pour l'acte de prélèvement - aux fins de don - de son sang $^{24}$, de tissus ${ }^{25}$ ou d'organes ${ }^{26}$. Certaines interdictions connaissent toutefois des exceptions. Le don du sang ${ }^{27}$ et le don de cellules hématopoiétiques issues de la moelle osseuse peuvent être entrepris dans des conditions très restrictives. Le législateur a envisagé la situation d'un enfant mineur en mesure de sauver la vie d'autrui, spécialement celle de son frère ou sa sœur ${ }^{28}$.

D'autre part, la loi permet aux personnes mineures de nouer une relation de confiance avec des médecins ou des infirmières sous le sceau du secret professionnel.

20. Voir, de manière générale, J.-J. Lemouland, «L'assistance du mineur, une voie possible entre l'autonomie et la représentation », Revue trimestrielle de droit civil, 1997, p. 1-24.

21. Voir l'affaire du Prozac où un médecin psychiatre a prescrit le médicament Prozac à une jeune fille mineure souffrant d'une «dépression modérée à sévère». Le médecin avait reçu une première fois la jeune adolescente avec son père et sa mère; mais elle était accompagnée seulement de sa mère lorsque le médecin a délivré cet antidépresseur. Le Conseil d'État a annulé la décision de la chambre nationale disciplinaire de l'ordre des médecins qui avait conclu à l'absence de manquement à la déontologie médicale. La prescription d'un antidépresseur n'était pas, en l'espèce, un acte usuel et requerrait le consentement des deux parents (CE, 7 mai 2014, n 359076: F. Vialla, "Prozac et acte usuel de l'autorité parentale», Revue droit et santé, n 63, 2015, p. 103; M. Beauruel, "Les actes usuels de l'autorité parentale ", in Les grandes décisions du droit des personnes et de la famille, A. Batteur (dir.), $2^{\mathrm{e}}$ éd., Issy-les-Moulineaux, LGDJ, 2016, p. 692-699).

22. Art. L. 2123-2, al. $1^{\text {er }}$ du Code de la santé publique: «La ligature des trompes ou des canaux déférents à visée contraceptive ne peut être pratiquée sur une personne mineure».

23. Art. L. 1244-2, al. 3 du Code de la santé publique: «Lorsqu'il est majeur, le donneur peut ne pas avoir procréé». Sur le prélèvement de gamètes aux fins de don à un couple et les personnes mineures ou majeures protégées, voir G. Raoul-Cormeil, «Une analyse contractuelle du don de gamètes ", in Le don de gamètes (Actes du colloque tenu à l'université d'Évry le 13 décembre 2012), A. Bertrand-Mirkovic (dir.), Bruxelles, Bruylant (Droit, bioéthique et société; 10), 2014, p. 27-48.

24. Art. L. 1221-5, al. $1^{\text {er }}$ du Code de la santé publique: «Aucun prélèvement de sang ou de ses composants en vue d'une utilisation thérapeutique pour autrui ne peut avoir lieu sur une personne mineure ou sur une personne majeure faisant l'objet d'une mesure de protection légale».

25. Art. L. 1241-2 du Code de la santé publique: «Aucun prélèvement de tissus ou de cellules, aucune collecte de produits du corps humain en vue de don ne peut avoir lieu sur une personne vivante mineure ou sur une personne vivante majeure faisant l'objet d'une mesure de protection légale».

26. Art. L. 1231-2 du Code de la santé publique: «Aucun prélèvement d'organes, en vue d'un don, ne peut avoir lieu sur une personne vivante mineure ou sur une personne vivante majeure faisant l'objet d'une mesure de protection légale».

27. Art. L. 1221-5, al. 2 du Code de la santé publique: "Toutefois, s'agissant des mineurs, un prélèvement peut être effectué à titre exceptionnel, lorsque des motifs tirés de l'urgence thérapeutique l'exigent ou lorsqu'il n'a pu être trouvé de donneur majeur immunologiquement compatible». La décision reposera donc sur une décision médicale mais chacun des parents et l'enfant mineur peuvent s'y opposer: «Le prélèvement ne peut alors être opéré qu'à la condition que chacun des titulaires de l'autorité parentale y consente expressément par écrit» (al. 3); «Le refus de la personne mineure fait obstacle au prélèvement» (al. 4).

28. Art. L. 1241-3, al. $1^{\text {er }}$ du Code de la santé publique: «Par dérogation aux dispositions de l'article L. 1241-2, en l'absence d'autre solution thérapeutique un prélèvement de cellules hématopoiétiques recueillies par prélèvement dans la moelle osseuse ou dans le sang périphérique peut être fait sur un mineur au bénéfice de son frère ou de sa sœur». Le texte comporte d'autres alinéas qui ajoutent d'autres dérogations exceptionnelles. 
La décision du législateur de traiter le mineur comme un patient autonome a été prise dans le contexte singulier de l'interruption volontaire de grossesse libéralisée par la loi du 4 juillet $2001^{29}$. L'autonomie du mineur a été généralisée par la loi «démocratie sanitaire» du 4 mars 2002 puis par la loi réformant notre système de santé promulguée le 26 janvier 2016. C'est dire que le médecin, la sage-femme ou l'infirmière peuvent aujourd'hui être dispensés par le mineur lui-même de l'obligation légale de rechercher l'autorisation des père et mère avant de prendre un acte médical ${ }^{30}$ ou infirmier ${ }^{31}$ sur le corps du patient mineur. Ces textes dérogent expressément aux dispositions de l'autorité parentale portées par le Code civil. Ils ont été analysés comme fondant une capacité spéciale médicale ou une prémajorité sanitaire ${ }^{32}$. On peut hésiter à partager une telle qualification car, en toute rigueur, la capacité juridique est étrangère à la volonté individuelle. Nul ne peut par son seul consentement créer une incapacité juridique ou se délivrer d'une incapacité juridique. Ces règles du Code de la santé publique sont d'une nature singulière. D'application exceptionnelle, ces règles traduisent le déclin de l'autorité parentale. Qu'en est-il maintenant de l'autorité tutélaire?

\section{B. Fondement et technique de la protection des majeurs vulnérables}

Pour les majeurs protégés, la loi du 5 mars 2007 a consacré la vocation à la plénitude de la protection ${ }^{33}$. Toute personne majeure qui souffre d'une altération de ses facultés mentales ou d'un empêchement physique d'exprimer sa volonté reçoit la protection judiciaire de sa personne et de ses biens. La protection de la personne obéit à un cadre juridique spécifique, distinct de la protection des biens. C'est là l'un des principaux apports de la réforme de la protection juridique des personnes majeures ${ }^{34}$. Le droit applicable à la protection de la personne a été conçu de manière générale et presque à droit constant suivant toutes les personnes majeures protégées. Ainsi, les articles 457-1 à 459-2 du Code civil ne sont pas réservés à la tutelle et à la curatelle; ils sont aussi applicables - par renvoi - à la sauvegarde de justice ${ }^{35}$, au mandat de protection future ${ }^{36}$ et à l'habilitation familiale ${ }^{37}$. En clair, la protection de la personne a été plutôt conçue de manière générale. À l'exception du mariage, du divorce et du pacte civil de solidarité, la loi civile ne distingue pas selon la nature de la mesure de protection juridique.

29. À propos de l'interruption volontaire de grossesse, voir l'article L. 2212-7, alinéa 3 du Code de la santé publique (loi nº $2001-588$ du 4 juillet 2001): «Dans ce cas, la mineure se fait accompagner dans sa démarche par la personne majeure de son choix». Voir aussi l'article L. 5134-1, alinéa 1 ${ }^{\text {er }}$ du Code de la santé publique (loi $\mathrm{n}^{\circ}$ 2001-588 du 4 juillet 2001), aux termes duquel le consentement des titulaires de l'autorité parentale n'est pas requis pour la prescription, la délivrance ou l'administration de contraceptifs aux personnes mineures. Enrichi par la loi $\mathrm{n}^{\circ} 2012-1404$ du 17 décembre 2012, ce texte oblige le personnel médical à s'assurer de «l'accompagnement psychologique» du mineur. Précisons que la prise en charge par la Sécurité sociale des moyens de contraception et leur délivrance aux mineurs sans autorisation parentale par les centres de planification familiale datent de 1974 (art. 2 de la loi no 74-1026 du 4 décembre 1974, Journal officiel de la République française, 5 décembre 1974, p. 12123).

30. Art. L. 1111-5, al. $1^{\text {er }}$ du Code de la santé publique (loi $n^{\circ}$ 2002-303 du 4 mars 2002, modifiée par la loi nº 2016-41 du 26 janvier 2016): «Par dérogation à l'article 371-1 du code civil, le médecin ou la sage-femme peut se dispenser d'obtenir le consentement du ou des titulaires de l'autorité parentale sur les décisions médicales à prendre lorsque l'action de prévention, le dépistage, le diagnostic, le traitement ou l'intervention s'impose pour sauvegarder la santé d'une personne mineure, dans le cas où cette dernière s'oppose expressément à la consultation du ou des titulaires de l'autorité parentale afin de garder le secret sur son état de santé. Toutefois, le médecin ou la sage-femme doit dans un premier temps s'efforcer d'obtenir le consentement du mineur à cette consultation. Dans le cas où le mineur maintient son opposition, le médecin ou la sage-femme peut mettre en œuvre l'action de prévention, le dépistage, le diagnostic, le traitement ou l'intervention. Dans ce cas, le mineur se fait accompagner d'une personne majeure de son choix». Et l'alinéa $2^{\text {nd }}$ ajoute: «Lorsqu'une personne mineure, dont les liens de famille sont rompus, bénéficie à titre personnel du remboursement des prestations en nature de l'assurance maladie et maternité et de la couverture complémentaire mise en place par la loi n 99-641 du 27 juillet 1999 portant création d'une couverture maladie universelle, son seul consentement est requis».

31. Art. L. 1111-5-1 du Code de la santé publique (loi nº 2016-41 du 26 janvier 2016): «Par dérogation à l'article 371-1 du Code civil, l'infirmier peut se dispenser d'obtenir le consentement du ou des titulaires de l'autorité parentale sur les décisions à prendre lorsque l'action de prévention, le dépistage ou le traitement s'impose pour sauvegarder la santé sexuelle et reproductive d'une personne mineure, dans le cas où cette dernière s'oppose expressément à la consultation du ou des titulaires de l'autorité parentale afin de garder le secret sur son état de santé. Toutefois, l'infirmier doit, dans un premier temps, s'efforcer d'obtenir le consentement du mineur à cette consultation. Dans le cas où le mineur maintient son opposition, l'infirmier peut mettre en œuvre l'action de prévention, le dépistage ou le traitement. Dans ce cas, le mineur se fait accompagner d'une personne majeure de son choix».

32. Voir F. Dekeuwer-Défossez, «L'autorité parentale à l'épreuve de la loi Kouchner», Revue générale de droit médical, $\mathrm{n}^{\circ}$ 12, 2004, p. 99-104, spéc. p. 102. Voir aussi G. Fauré, "Vers l'émergence d'une majorité sanitaire», in La loi du 4 mars 20o2: continuité ou nouveauté en droit médical?, G. Fauré (dir.), Paris, PUF (CEPRISCA), 2003, p. 101; C. Rey-Salmon, "Secret médical et personnes vulnérables: le cas du mineur», Recueil Dalloz, 2009, p. 2651; D. Vigneau, «L'autonomie du mineur en matière de santé», in La condition juridique du mineur. Aspects internes et internationaux, J.-J. Lemouland (dir.), Paris, Litec (Carré droit), 2004, p. 41; P. Bonfils, A. Gouttenoire, Droit des mineurs, $\mathrm{n}^{\circ}$ 1244 sq., p. 749-754; J. Poirret, La représentation légale du mineur..., $\mathrm{n}^{\circ} 398$.

33. Voir J. Hauser, «La vocation à la plénitude de la protection du majeur», in Le patrimoine de la personne protégée, J.-M. Plazy, G. Raoul-Cormeil (dir.), Paris, LexisNexis, 2015, étude 27, p. 369-382. Voir aussi T. Fossier, T. Verheyde, «Réforme des tutelles: la protection de la personne», L'actualité juridique. Famille, avril 2007, p. 160; T. Verheyde, «La protection de la personne du majeur protégé », L'actualité juridique. Famille, janvier 2009, p. 19; A. Batteur, «Majeurs protégés. Curatelle et tutelle. Effets personnels», Jurisclasseur Code civil, 2015, fasc. 10, art. 457-1 à 463.

34. Voir, sous l'empire de la loi du 3 janvier 1968, J. Hauser, «Réflexions sur la protection de la personne de l'incapable», in Mélanges offerts à Pierre Raynaud, Paris, Dalloz, 1985, p. 227. Voir aussi Cass., $1^{\text {re }}$ civ., 18 avril 1989, $\mathrm{n}^{\circ}$ 87-14.563, Bulletin civil $\mathrm{I}$, $\mathrm{n}^{\circ}$ 156: La semaine juridique, édition générale, 1989, II, 21467, note T. Fossier; Recueil Dalloz, 1989, jurispr., p. 493, note J. Massip; G. Raoul-Cormeil, «La protection de la personne du majeur protégé», in Les grandes décisions..., p. 416-425.

35. Art. 438 du Code civil.

36. Art. 479, al. $1^{\text {er }}$ du Code civil.

37. Art. 494-6, al. 3 du Code civil. Sur lequel, voir A. Batteur, «Habilitation familiale et protection de la personne du majeur protégé», Droit de la famille, 2016, étude 45, p. 33-36. 
Le principe est la sauvegarde de l'autonomie: le majeur protégé prend seul les décisions qui concernent sa personne si son état le permet. La capacité juridique du majeur protégé est donc, en principe, axée sur sa propre aptitude à délivrer un consentement lucide et éclairé. Le droit s'aligne sur le fait. Posée à l'alinéa $1^{\text {er }}$ de l'article 459, la règle connaît trois types d'exceptions.

Première exception. Tout d'abord, le juge des tutelles peut restreindre l'autonomie de la personne protégée en requérant l'assistance du curateur ou du tuteur ou en soumettant, exceptionnellement, et par décision spécialement motivée, un acte ou une série d'actes personnels à la représentation du tuteur. Le juge bénéficie donc d'un grand pouvoir: il peut choisir entre l'assistance ou la représentation ${ }^{38}$; il peut aussi déterminer l'étendue restreinte ou quasi générale du domaine de l'incapacité d'exercice en matière personnelle. La loi a cependant posé une limite absolue au juge. Certaines décisions personnelles ne peuvent faire l'objet d'une assistance ou d'une représentation; la personne en charge de la mesure est écartée de la prise de décision au risque que le majeur protégé ne puisse pas mettre en œuvre ses droits subjectifs. La loi a défini la catégorie des actes réputés strictement personnels et dressé une liste indicative ${ }^{39}$. L'article $458 \mathrm{du}$ Code civil ne vise aucun acte médical, compte tenu du besoin de sauvegarder la vie humaine.

Deuxième exception. Ensuite, l'article 459, alinéa 3 du Code civil élève une limite au pouvoir d'assistance ou de représentation de la personne en charge de la protection de la personne. Lorsque la décision porte atteinte à l'intégrité corporelle du majeur protégé, la personne en charge de la mesure ne peut assister ou représenter le majeur protégé sans y avoir été préalablement autorisée par le juge des tutelles. Ce texte a été conçu pour la matière médicale. Le législateur de 2007 n'a pas souhaité prendre en considération le risque consécutif à une intervention chirurgicale ${ }^{40}$ mais la certitude d'une atteinte grave. La loi subordonne donc la décision médicale d'amputation d'un membre ${ }^{41}$ ou d'ablation d'un organe ${ }^{42}$ à l'autorisation du juge des tutelles que le curateur ou le tuteur doit requérir.

Troisième exception. Enfin, il faut retrancher de cette présentation tous les actes médicaux qui font l'objet d'une procédure spécifique suivant le Code de la santé publique. D'un côté, la loi entend mieux protéger les personnes protégées que le Code civil en posant des incapacités spéciales de jouissance. Il en est ainsi du prélèvement de sang $^{43}$, de tissus ${ }^{44}$ ou d'organes ${ }^{45}$ aux fins de don entre vifs. L'interdiction est vécue par les intéressés comme une discrimination intolérable. De surcroît, la règle d'ordre public posée à l'article 444 du Code civil est délicate à faire respecter en pratique: les médecins ne sont pas rompus à la pratique notariale consistant à se faire délivrer un extrait d'acte de naissance sur lequel est mentionné l'existence du jugement ouvrant la mesure de protection juridique. Or, nul ne saurait se contenter d'une attestation de la personne déclarant ne pas être bénéficiaire d'une mesure de protection juridique. Par ailleurs, certains actes médicaux font l'objet d'une procédure spécifique plus protectrice que la simple assistance ou la représentation parce que la décision médicale doit être subordonnée à l'autorisation préalable d'un comité d'experts : tel est le cas en matière de recherche biomédicale ${ }^{46}$, de stérilisation à

38. L'article 459, alinéa 2 du Code civil subordonne l'octroi d'un pouvoir de représentation à l'ouverture d'une tutelle. Mais si le juge des tutelles a ouvert une habilitation familiale ou une sauvegarde de justice avec mandat spécial, est-il nécessaire de transformer ces deux mesures qui fonctionnent avec la technique de la représentation en une mesure de tutelle? On peut sérieusement en douter. Il en serait de même $a$ fortiori dans le cas du mandat de protection future. Il doit être possible pour le mandataire de solliciter l'autorisation du juge des tutelles de représenter le mandant en matière personnelle, sans qu'il soit nécessaire de mettre fin au mandat de protection future et d'ouvrir une tutelle!

39. Art. 458, al. $\mathbf{2}$ du Code civil: «Sont réputés strictement personnels la déclaration de naissance d'un enfant, sa reconnaissance, les actes de l'autorité parentale relatifs à la personne d'un enfant, la déclaration du choix ou du changement du nom d'un enfant et le consentement donné à sa propre adoption ou à celle de son enfant».

40. Voir, pour une coloscopie, TI Nice, ordonnance du juge des tutelles, 4 février 2009, no 08/00602: Dalloz, 2009, p. 1397, note T. Verheyde; Droit de la famille, 2009, comm. 147, note L. Talarico, p. 67; Revue trimestrielle de droit civil, 2010, p. 530, obs. J. Hauser. Voir aussi J. Massip, "Les règles applicables aux actes personnels et médicaux concernant un majeur en tutelle», Droit de la famille, 2010, étude 18, p. 18 , spéc. $\mathrm{n}^{\circ}$ 7.

41. Pour l'amputation d'une jambe, voir TI Caen, ordonnance du juge des tutelles, 26 juillet 2013, une décision complètement anonyme, sans rôle du greffe, que nous a transmise le service juridique de l'Association tutélaire du Calvados (ATC), autorisant l'amputation trans-fémorale de la jambe droite d'une personne en tutelle «dans l'intérêt de sa santé». On peut également citer l'arrachage de toutes les dents du majeur protégé avant la pose d'un appareil dentaire: TI Caen, ordonnance du juge des tutelles, 9 août 2012, une décision complètement anonyme, sans rôle du greffe, que nous a transmise le service juridique de l'Union départementale des associations familiales (UDAF) du Calvados.

42. Citons l'ablation d'un sein ou d'un rein (TI Caen, ordonnance du juge des tutelles, 17 mai 2010, RG nº $09 / 8520$, à propos d'une néphro-urétérectomie gauche) et l'intervention chirurgicale consistant en la pause d'une prothèse de hanche ou d'une poche gastrique (TI Caen, ordonnance du juge des tutelles, 28 février 2014, RG anonyme, cas de cancer du colon).

43. Art. L. 1221-5, al. $1^{\text {er }}$ du Code de la santé publique: «Aucun prélèvement de sang ou de ses composants en vue d'une utilisation thérapeutique pour autrui ne peut avoir lieu sur une personne mineure ou sur une personne majeure faisant l'objet d'une mesure de protection légale».

44. Art. L. 1241-2 du Code de la santé publique: «Aucun prélèvement de tissus ou de cellules, aucune collecte de produits du corps humain en vue de don ne peut avoir lieu sur une personne vivante mineure ou sur une personne vivante majeure faisant l'objet d'une mesure de protection légale».

45. Art. L. 1231-2 du Code de la santé publique: «Aucun prélèvement d'organes, en vue d'un don, ne peut avoir lieu sur une personne vivante mineure ou sur une personne vivante majeure faisant l'objet d'une mesure de protection légale».

46. En tutelle, art. L. 1122-2 II, al. 2 du Code de la santé publique: «Lorsqu'une recherche biomédicale est effectuée sur une personne mineure ou majeure sous tutelle, l'autorisation est donnée par son représentant légal et, si le comité mentionné à l'article L. 1123-1 considère que la recherche comporte, par l'importance des contraintes ou par la spécificité des interventions auxquelles elle conduit, un risque sérieux d'atteinte à la vie privée ou à l'intégrité du corps humain, par le conseil de famille s'il a été institué, ou par le juge des tutelles». En curatelle, art. L. 1122-2 II, al. 4 du Code de la santé publique: «Lorsqu'une recherche biomédicale est effectuée sur une personne majeure sous curatelle, le consentement est donné par l'intéressé assisté par son curateur. Toutefois, si la personne majeure sous curatelle est sollicitée en vue de sa participation à une recherche dont le comité mentionné à l'article L. 1123-1 considère qu'elle comporte, par l'importance des contraintes ou par la spécificité des 
visée contraceptive ${ }^{47}$ ou de prélèvement de cellules hématopoïétiques issues de la moelle osseuse ${ }^{48}$. En revanche, rien n'est prévu en matière d'assistance médicale à la procréation, ni à propos de l'acte d'interruption de grossesse pour un motif discrétionnaire ou médical, de sorte que la doctrine discute du point de savoir s'il s'agit d'actes strictement personnels ${ }^{49}$.

Cette présentation sommaire des règles du Code civil en matière de prise de décision médicale concernant le corps d'une personne majeure protégée peut paraître bien complexe. Le droit positif l'est à certains égards. Mais, à s'en tenir à l'essentiel, les trois règles de l'article $459 \mathrm{du}$ Code civil compose un système rationnel et gradué qui préserve l'autonomie de la personne majeure protégée. Les hypothèses d'assistance sont limitées aux décisions les plus graves. La représentation est même exceptionnelle et devrait être réservée aux cas où la personne est hors d'état de s'exprimer. En somme, le contrôle tutélaire demeure nécessaire et légitime dans l'ordre du droit civil. Le Code de la santé publique obéit toutefois à une autre logique où la décision est prise en responsabilité par le médecin. Et, même si la loi oblige le médecin à rechercher le consentement du patient, il lui est difficile d'accepter l'emprise d'un tiers sur le corps d'autrui.

\section{La dégradation du contrôle parental ou tutélaire dans l'ordre du Code de la santé publique}

Les médecins ignorent le Code civil; ils obéissent aux règles du Code de déontologie médicale dont la valeur est décrétale et donc inférieure à la loi. De surcroît, ni la partie législative, ni la partie réglementaire du Code de la santé publique n'est à jour de la protection graduée des personnes vulnérables mise en place par la loi du 5 mars 2007. Or, le Code de la santé publique ne respecte pas le cercle des personnes qui doivent consentir à un acte médical sur le corps d'une personne vulnérable (A). Ce droit spécialisé s'affranchit de la portée des refus et modifie même le paradigme de la décision médicale (B).

\section{A. L'élargissement du cercle des personnes ayant autorité}

De 1953 à 2002, le Code de la santé publique ne comportait que des règles spéciales. Et, même si leur caractère dérogatoire s'est accentué avec les lois récentes, le Code de la santé publique restait dans le sillage du Code civil. La loi du 4 mars 2002, dite « démocratie sanitaire»a opéré une rupture. Le législateur a introduit au seuil du Code de la santé publique des règles générales qui posent le cadre de la relation que le médecin doit entretenir avec la personne malade. Or, au sein de ces règles générales, on découvre des formulations approximatives d'une autre époque, comme en témoigne l'assimilation révolue de la personne mineure et de la personne majeure en tutelle. Positivement, la loi entérine la distinction entre le consentement et la capacité juridique. Le médecin doit d'abord rechercher le consentement des patients mineurs ou majeurs en tutelle; mais il ne peut se contenter de leur consentement. Il doit aussi rechercher le consentement des personnes qui exercent l'autorité parentale ou tutélaire ${ }^{50}$ et c'est à cette fin qu'il doit les informer de l'état de santé du patient mineur ou majeur en tutelle ${ }^{51}$. En clair, le médecin ne peut pas opposer le secret professionnel aux personnes qui exercent l'autorité parentale ou tutélaire. Le Code de la santé publique aurait dû être cependant plus précis et distinguer selon que la personne vulnérable mineure ou en tutelle peut consentir ou ne le peut pas parce qu'elle est immature ou inconsciente. La mutation du mécanisme de l'assistance en représentation n'est pas anodine, ni pour les personnes mineures ni pour les personnes majeures vulnérables. En toute rigueur, elle

interventions auxquelles elle conduit, un risque sérieux d'atteinte à la vie privée ou à l'intégrité du corps humain, le juge des tutelles est saisi aux fins de s'assurer de l'aptitude à consentir du majeur. En cas d'inaptitude, le juge prend la décision d'autoriser ou non la recherche biomédicale».

47. En curatelle comme en tutelle, art. L. 2123-2, al. $1^{\text {er }}$ du Code de la santé publique: «La ligature des trompes ou des canaux déférents à visée contraceptive $[. .$.$] ne peut être pratiquée sur une personne majeure dont l'altération des facultés mentales constitue un handicap et a justifié$ son placement sous tutelle ou sous curatelle que lorsqu'il existe une contre-indication médicale absolue aux méthodes de contraception ou une impossibilité avérée de les mettre en œuvre efficacement».

48. En curatelle comme en tutelle, art. L. 1241-4, al. $1^{\text {er }}$ du Code de la santé publique: «Par dérogation aux dispositions de l'article L. 1241-2, en l'absence d'autre solution thérapeutique, un prélèvement de cellules hématopoiétiques issues de la moelle osseuse peut être fait sur une personne vivante majeure faisant l'objet d'une mesure de protection légale au bénéfice de son frère ou de sa sœur». Et l'alinéa 5 d'ajouter: "Avant de formuler l'avis mentionné au deuxième alinéa ou de délivrer les autorisations prévues aux troisième et quatrième alinéas, le comité d'experts mentionné à l'article L. 1231-3 s'assure que tous les moyens ont été mis en œuvre pour trouver un donneur majeur compatible pour le receveur». Les autres aliénas précisent la nature et l'étendue de l'incapacité d'exercice.

49. Voir, parmi les analyses proposées, A. Batteur, «Recherche d'une articulation entre le Code de la santé publique et le Code civil: un défi à relever en faveur des personnes vulnérables", Droit de la famille, 2011, étude 5, p. 22-27. Voir aussi G. Raoul-Cormeil, «Les incapacités médicales (petit guide pour une réécriture du Code de la santé publique)», in Mélanges en l'honneur de Gérard Mémeteau. Droit médical et éthique médicale: regards contemporains, Paris, Les études hospitalières, 2015, t. II, p. 109-127.

50. Art. L. 1111-4, al. 7 du Code de la santé publique: «Le consentement du mineur ou du majeur sous tutelle doit être systématiquement recherché s'il est apte à exprimer sa volonté et à participer à la décision. Dans le cas où le refus d'un traitement par la personne titulaire de l'autorité parentale ou par le tuteur risque d'entraîner des conséquences graves pour la santé du mineur ou du majeur sous tutelle, le médecin délivre les soins indispensables ».

51. Art. L. 1111-2, al. 5 du Code de la santé publique: «Les droits des mineurs ou des majeurs sous tutelle mentionnés au présent article sont exercés, selon les cas, par les titulaires de l'autorité parentale ou par le tuteur. Ceux-ci reçoivent l'information prévue par le présent article, sous réserve des dispositions de l'article L. 1111-5. Les intéressés ont le droit de recevoir eux-mêmes une information et de participer à la prise de décision les concernant, d'une manière adaptée soit à leur degré de maturité s'agissant des mineurs, soit à leurs facultés de discernement s'agissant des majeurs sous tutelle». 
suppose une décision du juge des tutelles et l'ouverture de la tutelle conformément à l'article 459, alinéa $2 \mathrm{du}$ Code civil. La distinction a échappé aux rédacteurs du Code de la santé publique qui ne s'embarrassent pas de la technique civiliste, ainsi que le montre l'élargissement du cercle des personnes ayant autorité pour participer à la décision médicale.

L'élargissement du cercle des personnes ayant autorité concerne d'abord les personnes majeures vulnérables. A priori, le Code de la santé publique ne vise que la personne majeure en tutelle dans ses articles L. 1111-2, alinéa 7 et L. 1111-4, alinéa 5. Seul le tuteur d'une personne majeure est destinataire des informations médicales concernant la personne en tutelle et, ainsi, invité à consentir à ses côtés ou à sa place si elle est inconsciente. Le Code de la santé publique considère donc, sauf exception légale expresse $^{52}$, que les autres personnes majeures placées sous mesure de protection juridique sont autonomes. Ainsi, les personnes placées en sauvegarde de justice ou en curatelle peuvent seules consentir valablement à tout acte médical les concernant. Il en est de même a fortiori du mandat de protection future dont la prise d'effet ne s'accompagne pas d'une incapacité juridique faute de jugement homologuant le contrat. Mais qu'en est-il lorsque le Code de la santé publique vise non pas le tuteur mais le représentant légal? En toute rigueur, la notion de représentant légal n'épouse que la situation des père et mère auxquels la loi attribue un pouvoir de représentation lorsqu'ils exercent l'autorité parentale. Le tuteur d'une personne majeure est désigné par un juge des tutelles; il tient son pouvoir d'un jugement et non pas de la loi, en vertu d'une vérification concrète de son aptitude à prendre en charge l'intérêt d'autrui. Les rédacteurs du Code de la santé publique ont jugé commode l'usage du vocable «représentant légal» car il reflétait l'assimilation du mineur et de la personne majeure en tutelle. La loi du 5 mars 2007 n'a pas négligé l'usage de cette notion : l'article 459-1 du Code civil affirme même la prévalence des règles dérogatoires du Code de la santé publique prévoyant l'intervention du représentant légal ${ }^{53}$. Cependant, à lire tout le Code de la santé publique, on s'aperçoit que le représentant légal désigne parfois le curateur ${ }^{54}$. Un magistrat spécialiste de droit des majeurs protégés reconnaissait la plasticité de cette expression et déplorait les incertitudes pratiques qu'elle engendrait ${ }^{55}$. Dans ces conditions, il est logique de soutenir que le représentant légal devrait aussi viser la personne habilitée à représenter la personne protégée par une mesure d'habilitation familiale ${ }^{56}$. En effet, alors que la personne en curatelle est dans une situation moins grave que la personne en tutelle, puisqu'elle n'a pas besoin d'être représentée mais assistée ou contrôlée dans les actes importants de sa vie civile ${ }^{57}$, la personne éligible à l'habilitation familiale doit être hors d'état de manifester sa volonté ${ }^{8}$. En revanche, le curateur n'est pas un représentant légal. La qualification est à proprement parler abusive. À ce stade néanmoins, il convient de conclure que la loi se méfie des personnes protégées et invite systématiquement la personne en charge de la protection à prendre part à l'information du patient pour donner son avis. En pratique, les mandataires judiciaires à la protection des majeurs ${ }^{59}$ auxquels sont attribuées les charges curatélaires ou tutélaires ont contracté l'usage de s'en remettre à la volonté du patient lorsqu'il est en état de manifester sa volonté, se conformant ainsi au principe de recherche d'autonomie posé à l'article 415, alinéa $3 \mathrm{du}$ Code civil.

L'élargissement du cercle des personnes ayant autorité concerne ensuite les personnes mineures. La rigueur du droit civil devrait obliger le médecin à requérir le consentement des seuls père et mère qui exercent l'autorité parentale. En pratique, la séparation des père et mère n'a plus d'incidence sur l'exercice conjoint de l'autorité parentale.

52. Parmi les textes spéciaux, citons l'interdiction de faire don de son sang (art. L. 1221-5 du Code de la santé publique) ou de s'engager dans un protocole de recherche biomédicale (art. L. 1122-2, II du Code de la santé publique). Dans le premier texte, la loi vise de manière générale toute personne bénéficiant d'une mesure de protection légale, ce qui embrasse la tutelle, la curatelle, la sauvegarde de justice et l'habilitation familiale qui s'ouvrent, toutes, par un jugement du juge des tutelles. Dans le second texte relatif à la recherche biomédicale, la loi vise distinctement la curatelle et la tutelle.

53. Art. 459-1, al. $1^{\text {er }}$ du Code civil: «L'application de la présente sous-section ne peut avoir pour effet de déroger aux dispositions particulières prévues par le Code de la santé publique et le Code de l'action sociale et des familles prévoyant l'intervention d'un représentant légal».

54. Art. L. 2123-2, al. 2 du Code de la santé publique: "L'intervention est subordonnée à une décision du juge des tutelles saisi par la personne concernée, les père et mère ou le représentant légal de la personne concernée». Or, l'alinéa $1^{\text {er }}$ qui précède vise les personnes majeures placées en tutelle et en curatelle.

55. T. Verheyde, «Les personnes majeures en fin de vie bénéficiant d'un régime de protection juridique. Analyse pratique», in Éthique et conditions de la fin de vie, A. Batteur, G. Raoul-Cormeil (dir.), Paris, Mare \& Martin, 2016, étude 17, p. 197-203, spéc. p. 199: «Le représentant légal, pour un juge des tutelles, pour un mandataire judiciaire à la protection des majeurs, cela ne peut pas être quelqu'un d'autre qu'un tuteur. On a évoqué tout à l'heure la distinction fondamentale assistance/représentation; un représentant légal ne peut être, normalement, qu'un tuteur. Or les rédacteurs du Code de la santé publique se trouvent plutôt du côté du ministère de la santé que du ministère de la justice, lui-même en charge de la rédaction des textes du texte civil. Quand les rédacteurs du ministère de la santé ont écrit "représentant légal", ils avaient certainement en tête: personne chargée de la mesure de protection, tutelle ou curatelle. Mais, du simple fait de cette question de vocabulaire, il y a des hésitations et des controverses sur l'interprétation de ces dispositions - les juristes aiment bien les controverses d'une manière générale, même quand c'est relativement clair; alors si cela en plus n'est pas clair, cela complique encore plus les choses pour les praticiens! - mais cela a des effets très concrets: tout le monde n'est pas d'accord sur ce qu'il faut faire ou ne pas faire pour un certain nombre d'actes médicaux...».

56. Voir A. Batteur, «Habilitation familiale...».

57. Art. 440 du Code civil.

58. Art. 494-1 du Code civil.

59. Voir J. Bouton, «Les mandataires judiciaires à la protection des majeurs et la santé de la personne vulnérable», in Mélanges en l'honneur de Gérard Mémeteau..., p. 293-304. 
Le principe dit de coparentalité connaît aujourd'hui un large domaine d'application. Mais les textes du Code de la santé publique font preuve d'une souplesse insoupçonnée; ils ne distinguent pas entre les parents qui exercent l'autorité parentale et ceux qui en sont seulement titulaires sans pouvoir pleinement l'exercer. En clair, le parent qui n'exerce plus l'autorité parentale sur son enfant mineur et ne bénéficie que d'un droit de visite et d'hébergement ou seulement d'un droit de visite doit être consulté par le médecin comme s'il continuait à exercer l'autorité parentale. Le Code de la santé publique est ici aussi très approximatif dans sa partie législative ${ }^{60}$, comme dans sa partie réglementaire qui abrite le Code de déontologie médicale ${ }^{61}$. L'élargissement du cercle des personnes ayant autorité est l'une des manifestations du relâchement de la valeur du consentement des personnes ayant autorité.

\section{B. Le relâchement de la valeur de leur consentement}

L'élargissement du cercle des personnes habilitées à être consultées par les médecins est un leurre. D’autres textes du Code de la santé publique tendent à montrer que la loi entend éviter au parent ou au tuteur de prendre des décisions graves. On ne fera qu'évoquer la loi du 4 mars 2002 ayant consacré la jurisprudence du Conseil d'État rendue en matière de refus de transfusion sanguine par les parents témoins de Jéhovah ${ }^{62}$. Le médecin peut passer outre le refus du parent ou du tuteur lorsqu'il aggrave l'état de santé de la personne vulnérable; le médecin est alors habilité par la loi à prendre toute décision médicale pour sauvegarder la vie du patient. On peut évidemment se réjouir de cette solution mais elle témoigne que le médecin est en charge de l'intérêt du patient au même titre que celles et ceux qui exercent une autorité parentale ou tutélaire.

La fin de vie d'un mineur ${ }^{63}$ ou d'un majeur en tutelle illustre mieux le relâchement de la valeur du consentement de l'autorité parentale ou tutélaire. Dans ce contexte douloureux, le Code de déontologie médicale n'évoque plus le consentement des parents ou du tuteur. Il oblige seulement le médecin à solliciter leur avis ${ }^{64}$, au même titre que celui des autres membres de la famille. L'avis est un terme choisi. Le Code de la santé publique ne parle ni de consentement ni d'autorisation. L'enjeu de la limitation ou de l'arrêt des traitements marque une rupture avec le paradigme de la codécision médicale. Ce n'est plus au patient de prendre la décision avec le professionnel de santé mais au médecin de prendre la décision de limitation ou d'arrêt des traitements ${ }^{65}$. Le pouvoir médical est plus grand lorsque le patient est vulnérable; le médecin ne peut pas s'abriter derrière un refus de soins du patient. Mais faut-il se résoudre à admettre que le pouvoir de l'autorité parentale ou tutélaire se réduit à un avis? Rien n'est moins sûr.

Dans l'arrêt Lambert, la Cour de cassation a rappelé le cadre légal dans lequel le tuteur devra exercer ses prérogatives. Rappelons que Vincent Lambert est en état pauci-relationnel ou de conscience minimale depuis le grave accident de la circulation dont il a été victime le 20 septembre 2008. Après le Conseil d'État ${ }^{66}$ et la Cour

60. Art. L. 1111-4, al. 5 du Code de la santé publique: «Le consentement du mineur ou du majeur sous tutelle doit être systématiquement recherché s'il est apte à exprimer sa volonté et à participer à la décision. Dans le cas où le refus d'un traitement par la personne titulaire de l'autorité parentale ou par le tuteur risque d'entraîner des conséquences graves pour la santé du mineur ou du majeur sous tutelle, le médecin délivre les soins indispensables" (nous soulignons).

61. Art. R. 4127-42 du Code de la santé publique: «Sous réserve des dispositions de l'article L. 1111-5, un médecin appelé à donner des soins à un mineur ou à un majeur protégé doit s'efforcer de prévenir ses parents ou son représentant légal et d'obtenir leur consentement» (nous soulignons).

62. CE, Ass., 26 octobre 2001, Catherine Senayake: Droit et patrimoine, $\mathrm{n}^{\circ}$ 101, 2002, p. 111, obs. G. Loiseau; Revue de droit sanitaire et social, 2002, p. 41-49, note L. Dubouis; Revue française de droit administratif, 2002, p. 146-162, concl. D. Chauvaux, note D. de Béchillon; Revue trimestrielle de droit civil, 2002, p. 484, obs. J. Hauser. CE, réf., 16 août 2002, Valérie et Isabelle Feuillatey: Droit et patrimoine, ${ }^{\circ}{ }^{110}$, 2002, p. 84, obs. G. Loiseau; La semaine juridique, édition générale, 2002, II, 10084, note P. Mistretta; Revue trimestrielle de droit civil, 2002, p. 781 sq., obs. J. Hauser.

63. Voir, par exemple, CE, 8 mars 2017, $\mathrm{n}^{\circ}$ 408146, Recueil Dalloz, 2017, p. 574: Droit de la famille, 2017, comm. 114, p. 64-66, note A. Mirkovic, où les magistrats du Palais-Royal ont jugé que les soins prodigués à un enfant polyhandicapé sous assistance respiratoire et alimentation artificielle ne relèvent pas de l'obstination déraisonnable, contrairement à la décision du médecin en charge de l'enfant de 10 mois. Il est regrettable que la décision du Conseil d'État n'ait pas cherché à justifier la décision autrement que par les règles du Code de la santé publique. Les père et mère qui exercent l'autorité parentale représentent leur enfant; ils n'ont pas à être sollicités pour ne délivrer qu'un avis dans le cadre de la procédure collégiale. Pour aller plus loin, voir A. Batteur, «La fin de vie des mineurs en France. Analyse juridique», in Éthique et conditions de la fin de vie, étude 20, p. 221-235.

64. Art. R. 4127-37-2, II, al. 2 du Code de la santé publique: «Lorsque la décision de limitation ou d'arrêt de traitement concerne un mineur ou un majeur protégé, le médecin recueille en outre l'avis des titulaires de l'autorité parentale ou du tuteur, selon les cas, hormis les situations où l'urgence rend impossible cette consultation" (nous soulignons).

65. Art. L. 1110-5-1 à L. 1110-5-3 du Code de la santé publique. Sur le nouveau dispositif issu de la loi Clayes et Léonetti, voir P. Mistretta, «De l’art de légiférer avec tact et mesure», La semaine juridique, édition générale, 2016, doctr. 240; A. Denizot, "Le nouveau droit de la fin de vie», Revue trimestrielle de droit civil, 2016, p. 460; R. Desgorces, «La loi nº 2016-87 du 2 février 2016 créant de nouveaux droits en faveur des malades et des personnes en fin de vie", Petites affiches, $\mathrm{n}^{\circ}$ 117, 13 juin 2016; J.-R. Binet, "Présentation de la loi créant de nouveaux droits en faveur des malades et des personnes en fin de vie», Droit de la famille, 2016, dossier 34, p. 15-18.

66. CE, Ass., 24 juin 2014, $\mathrm{n}^{\circ} 375081, \mathrm{n}^{\circ} 375090, \mathrm{n}^{\circ} 375091$ : L'actualité juridique. Droit administratif, 2014, p. 1669, note D. Truchet; Recueil Dalloz, 2014, p. 1856, note D. Vigneau; Revue française de droit administratif, 2014, p. 666 et p. 657-665, concl. R. Keller; Droit de la famille, 2014, comm. 141, obs. J.-R. Binet; La semaine juridique, édition générale, 2014, p. 825, obs. F. Vialla; Médecine et droit, 2014, p. 129, note G. Mémeteau; Revue de droit sanitaire et social, 2014, p. 1101, note D. Thouvenin; Revue de droit de la santé, n 61, 2014, p. 118, obs. B. Legros. Voir aussi P. Delvolvé, "Glissements», Revue française de droit administratif, 2014, p. 702-710; M. Canedo-Paris, «Le juge administratif et l'euthanasie: les apports de l'affaire Vincent Lambert", Revue du droit public, n' 1, 2015, p. 1-82; J.-M. Larralde, "L'arrêt des soins prodigués à un patient inconscient: observations sur l'arrêt du Conseil d'État, Vincent Lambert ", in Éthique et conditions de la fin de vie, étude 13, p. 159-168. 
européenne des droits de l'homme ${ }^{67}$, c'est la Cour de cassation qui a été saisie d'un pourvoi dont l'objet fut de critiquer notamment le bien-fondé de l'ouverture d'une tutelle au profit de Vincent Lambert. Selon l'arrêt de rejet,

[...] il était nécessaire de désigner un représentant légal, afin que [le patient hors d'état de s'exprimer] soit représenté dans les différentes procédures le concernant et que les décisions relatives à sa personne puissent être prises dans son seul intérêt, sous le contrôle du juge des tutelles, conformément aux dispositions de l'article 459 du code civil, sans préjudice des dispositions du code de la santé publique applicables $[\ldots]^{68}$.

La Cour de cassation a eu raison de citer d'abord l'article 459 du Code civil. Selon l'alinéa $1^{\text {er }}$ de ce texte, «la personne protégée prend seule les décisions relatives à sa personne dans la mesure où son état le permet ". Puis l'alinéa 2 ajoute que le juge peut, lorsque l'état de la personne protégée ne lui permet pas de prendre seule une décision personnelle éclairée, autoriser le tuteur à représenter l'intéressé. C'est dire qu'en principe le tuteur ne représente pas de manière générale la personne protégée pour prendre, à sa place et dans son intérêt, des décisions personnelles. Le tuteur doit être habilité, acte par acte, par le juge des tutelles, à moins qu'il n'ait reçu un pouvoir général de représentation en matière d'actes personnels. Même en ce cas, le pouvoir général de représentation du tuteur connaît des limites qui sont de deux ordres. D'une part, le tuteur ne peut pas prendre à la place du majeur protégé des décisions strictement personnelles au sens de l'article 458 du Code civil. Or, il nous semble plus que douteux que l'arrêt des traitements relève de cette catégorie juridique car ni l'avis du tuteur, ni l'avis de la famille n'aurait besoin, en cas d'inconscience du majeur protégé, d'être sollicité par les médecins. La représentation du tuteur est donc une technique juridique nécessaire pour prendre avec le médecin la décision de limitation ou d'arrêt des traitements.
D'autre part, la loi no 2007-308 du 5 mars 2007 a limité le pouvoir du tuteur lorsque la décision médicale est de nature à porter une grave atteinte à l'intégrité corporelle du majeur protégé. Il faut ici raisonner suivant l'argument a fortiori. Puisque le tuteur doit solliciter l'autorisation du juge des tutelles pour représenter le majeur protégé inconscient à l'acte médical d'amputation d'un membre ou d'ablation d'un organe, alors c'est à plus forte raison qu'il doit solliciter cette autorisation lorsque la vie du majeur protégé est en jeu par la décision médicale. L'exercice du pouvoir de représentation est, nous semble-t-il, soumis, hors le cas de l'urgence médicale, à l'autorisation du juge des tutelles exigée par l'article 459, alinéa 3 du Code civil. On ne saurait donc dire que le médecin doit se contenter d'un avis du tuteur.

Au terme de cette présentation des règles gouvernant l'intervention de l'autorité parentale ou tutélaire dans le processus de soin des personnes mineures ou majeures protégées, il faut conclure que la pratique médicale triomphe presque toujours de l'obscurité de la loi. On pourrait souhaiter une réécriture du Code de la santé publique et du Code civil dans le but de rétablir la cohérence entre les textes $^{69} \ldots$ mais l'essentiel est aujourd'hui dans la formation des médecins à l'éthique médicale. Éclairés par des discussions collégiales et des débats pluridisciplinaires, les médecins continueront à prendre seuls les décisions médicales les plus graves car ils en portent la responsabilité. Les père et mère ou les tuteurs devront être associés à ces décisions sans que leur parole soit tenue pour égale à l'avis des autres membres de la famille. Si le dialogue a pu se nouer entre le médecin, le patient vulnérable lorsqu'il peut s'exprimer, ses parents ou la personne en charge de sa protection, et c'est le cas dans l'immense majorité des cas, alors les personnes exerçant l'autorité parentale ou tutélaire seront associées à la bonne décision et n'auront donc pas à former de recours devant le juge compétent pour faire appliquer, en toute rigueur, la loi du Code civil.

67. Cour EDH, GC, 5 juin 2015, no 46043/14: Dalloz, 2015, p. 1625, note F. Vialla; Droit de la famille, 2015, comm. 180, obs. J.-R. Binet; La semaine juridique, édition générale, 2015, p. 805 et 1331, obs. F. Sudre; Revue trimestrielle des droits de l'homme, 2015, p. 1097, note J.-M. Larralde. Voir aussi G. Mémeteau, «Traversée civiliste du couloir de la mort», Gazette du Palais, nº 246, 2-3 septembre 2015, p. 7; J.-M. Larralde, «L'aide à mourir dans la jurisprudence de la Cour européenne des droits de l'homme: de Diane Pretty à Vincent Lambert», in Éthique et conditions de la fin de vie, étude 12, p. 145-157.

68. Cass., $1^{\text {re }}$ civ., 8 décembre 2016, $\mathrm{n}^{\mathrm{o}}$ 16-20.298, JurisData, $\mathrm{n}^{\circ}$ 2016-025731: Recueil Dalloz, 2016, p. 2569, obs. F. Vialla et 2017, p. 1500, obs. J.-J. Lemouland La semaine juridique, édition générale, 2017, p. 79, note J. Hauser; Droit de la famille, 2017, comm. 48, p. 52-54, note I. Maria; Revue générale de droit médical, $\mathrm{n}^{\circ} 62,2017$, p. 133-157, note G. Raoul-Cormeil.

69. Pour preuve, citons l'aveu du législateur et sa commande faite au gouvernement. Ainsi, selon l'article 211 de la loi no $2016-41$ du 26 janvier 2016 de modernisation de notre système de santé (Journal officiel de la République française, no o022, 27 janvier 2016), «Dans les conditions prévues à l'article 38 de la Constitution, le Gouvernement est autorisé à prendre par ordonnances, dans un délai de dix-huit mois à compter de la promulgation de la présente loi, les mesures relevant du domaine de la loi permettant de mieux articuler les dispositions du code civil et du code de la santé publique relatives aux conditions dans lesquelles peut s'exprimer la volonté des personnes faisant l'objet d'une mesure de protection juridique, au sens du chapitre II du titre XI du livre I ${ }^{e r}$ du code civil, pour toute décision relative à un acte médical. Un projet de loi de ratification est déposé devant le Parlement dans un délai de six mois à compter de la publication de l'ordonnance» (nous soulignons). Tempus fugit... Rien n'avait encore été entrepris le 15 mai 2017 ! Le contexte politique, marqué par la fin du quinquennat, la campagne électorale de l'élection présidentielle et la mise en œuvre des réformes promises, aura précipité la caducité de cette autorisation. Le 26 juillet 2017 , la direction des affaires civiles et du sceau de la chancellerie a expliqué aux représentants des mandataires judiciaires à la protection des majeurs pourquoi l'ordonnance n'avait pas été publiée: l'objet de l'habilitation n'était pas assez large et ne permettait pas d'articuler les pouvoirs de la personne en charge de la mesure de protection juridique en matière de santé avec ceux de la personne de confiance. Le gouvernement devrait donc solliciter une nouvelle demande d'habilitation au Parlement... 